\title{
Graphene-electrode array for brain map remodeling of the cortical surface
}

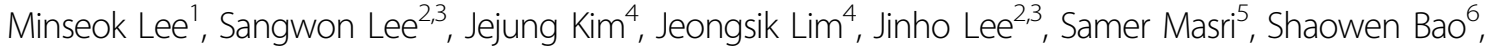 \\ Sunggu Yang $\mathbb{E}^{2}$, Jong-Hyun Ahn $\mathbb{E}^{4}$ and Sungchil Yang $\mathbb{1}^{1}$
}

\begin{abstract}
Cortical maps, which are indicative of cognitive status, are shaped by the organism's experience. Previous mapping tools, such as penetrating electrodes and imaging techniques, are limited in their ability to be used to assess highresolution brain maps largely owing to their invasiveness and poor spatiotemporal resolution, respectively. In this study, we developed a flexible graphene-based multichannel electrode array for electrocorticography (ECoG) recording, which enabled us to assess cortical maps in a time- and labor-efficient manner. The flexible electrode array, formed by chemical vapor deposition (CVD)-grown graphene, provided low impedance and electrical noise because a good interface between the graphene and brain tissue was created, which improved the detectability of neural signals. Furthermore, cortical map remodeling was induced upon electrical stimulation at the cortical surface through a subset of graphene spots. This result demonstrated the macroscale plasticity of cortical maps, suggesting perceptual enhancement via electrical rehabilitation at the cortical surface.
\end{abstract}

\section{Introduction}

Cortical sensory maps reflect the spatial organization of neural networks representing sensorimotor behavior and cognition and provide an accessible measure of cognitive learning enforced by experience. Cortical maps are not permanently fixed after their formation during the early developmental stage; they undergo structural and functional changes throughout an organism's lifespan as a consequence of interactions between adaptation and stabilization processes ${ }^{1-3}$. Cortical maps are sculpted by passive-stimulus exposure (e.g., environmental sensory exposure), reinforcement-based tasks (e.g., perceptual learning and reward-based demand), and traumatic experiences (e.g., distal deafferentation $)^{4-9}$.

Correspondence: Sunggu Yang (sungguyang@inu.ac.kr) or JongHyun Ahn (ahnj@yonsei.ac.kr) or Sungchil Yang (sungchil.yang@cityu.edu.hk) ${ }^{1}$ Department of Neuroscience, City University of Hong Kong, Tat Chee Avenue, Kowloon, Hong Kong

2Department of Nanobioengineering, Incheon National University, Incheon 22012, Republic of Korea

Full list of author information is available at the end of the article

These authors contributed equally: Minseok Lee, Sangwon Lee, Jejung Kim
Our study and other previous studies have focused on identifying cellular mechanisms that mediate sensorymap plasticity driven by Hebbian synaptic plasticity ${ }^{1,10-12}$. To date, many questions concerning sensory map plasticity remain unanswered because of the technical difficulty encountered in a quick assessment of the entire area of a cortical map to detect changes over time.

The use of penetrating electrodes or liquid-filled glass micropipettes to acquire decent brain maps for brain mapping requires tremendous amounts of labor and time. In addition, these techniques are invasive and damage brain tissue, thus preventing repetition ${ }^{13}$. Alternative brain-mapping tools, such as electroencephalography, positron-emission tomography, magnetoencephalography, and functional magnetic resonance imaging (fMRI), are noninvasive, enabling whole-brain mapping with repeated sampling. These methods are being increasingly used for brain mapping; however, they have disadvantages, such as low spatial resolution, temporal lag, errors due to unspecified modulatory inputs, and/or prohibitive $\operatorname{costs}^{14-16}$. Other options include optical techniques, such as calcium imaging and optogenetic

\section{(c) The Author(s) 2021}

(c) (i) Open Access This article is licensed under a Creative Commons Attribution 4.0 International License, which permits use, sharing, adaptation, distribution and reproduction c. in any medium or format, as long as you give appropriate credit to the original author(s) and the source, provide a link to the Creative Commons license, and indicate if changes were made. The images or other third party material in this article are included in the article's Creative Commons license, unless indicated otherwise in a credit line to the material. If material is not included in the article's Creative Commons license and your intended use is not permitted by statutory regulation or exceeds the permitted use, you will need to obtain permission directly from the copyright holder. To view a copy of this license, visit http://creativecommons.org/licenses/by/4.0/. 
fMRI. These techniques enable large-scale brain mapping but cannot offer clinical benefits owing to indicator (or viral) toxicity and/or low spatiotemporal resolution ${ }^{17,18}$.

As an alternative method, flexible surface electrodes composed of thin metal films or conducting polymers have attracted considerable attention owing to their high recording fidelity ${ }^{19-21}$. Recently, research has been conducted on the application of atomically thin graphene to surface multichannel electrodes for electrocorticography (ECoG) recording. Graphene integrated into an ECoG array exhibits good mechanical flexibility, long-term biocompatibility, and a high signal-to-noise ratio (SNR), allowing for large-scale real-time stable recordings even in a convoluted brain ${ }^{21-24}$.

Herein, we propose a graphene-based surface electrode array for investigating cortical maps and their plasticity. The microfabricated graphene array was placed on the cortical surface for the detection and induction of brain signals. We found that somatosensory and auditory maps could be rapidly constructed using the graphene array. Furthermore, cortical-surface stimulation through a subset of graphene electrodes could enhance and expand sensory responses. This graphene technology could provide new therapeutic interventions for various sensory and cortical disorders.

\section{Results}

Thirty-two or 128 multichannel graphene arrays were built to precisely detect neural signals on the cortical surface in response to auditory and somatosensory stimuli, respectively (Fig. 1a and Figs. S1-2). The incorporation of thru-holes of the device substrate between all electrodes allowed significantly stronger contact with the brain surface and the simultaneous use with penetrating electrodes (Fig. 1b and Fig. S1). Atomically thin graphene, which is composed of sp2-bonded carbon groups, can achieve good bonding and conformal contact with the skin, resulting in low electrical impedance and a high SNR. In particular, four-layer $(4 \mathrm{~L})$ graphene was used because it could provide low impedance for better detection of ECoG signals.

Figure 1c, d shows a Nyquist plot and an impedance histogram, respectively, of $4 \mathrm{~L}$-doped graphene and gold electrodes with the same electrode design. The calculated results of parameters in the equivalent circuit model were made using EIS measurements in phosphate-buffered saline (Table S1). The impedance of a graphene electrode is approximately 50 times lower than that of a gold electrode. The low impedance can suppress electrical noise, allowing the electrode size to be scaled down and increasing the detectability of neural signals through a high SNR. With these excellent characteristics of graphene multichannel electrodes for measuring neural signals, we studied cortical map plasticity over an area of several square millimeters. To investigate the mechanical stability of the graphene electrode array on a nonplanar surface, we measured the impedance changes of graphene electrodes with respect to the bending radius (Fig. 1e). With a small bending radius $(5 \mathrm{~mm})$, the graphene electrodes still maintained their impedance value below $100 \mathrm{k} \Omega$. These results indicated that the graphene electrode array could conduct uniform spatial brain mapping with low noise on a nonplanar cortical surface.

Figure 1f presents illustrations of a rat's auditory and somatosensory cortices and the shape of the rat's body, as presented in actual proportions on a somatosensory cortex map. Auditory stimuli with various frequency tones and physical stimuli to body parts, such as the whiskers, trunk, limbs, and paws, highlighted by different colors, induced location-dependent neural responses (evident on both auditory and somatosensory cortex maps). With the graphene array situated on the cortical surface, we recorded stimulus-specific responses on multiple channels (Fig. 1g). Each colored box highlighted in the 30 channel recording results is closely correlated with the geographical location of the somatosensory map of the rat.

We assessed the sensory maps by recording cortical responses to sensory stimuli using either a graphenesurface array or penetrating electrodes. We compared layer-specific cortical maps for two main reasons: first, to elucidate how information changes from the thalamorecipient layer (or layer 4) to the cortical surface, and second, to compare the response quality of graphene-surface recordings to that of penetrating layer 4 recordings.

Pure-tone pips $\left(50 \mathrm{~ms}, 5-\mathrm{ms} \cos ^{2}\right.$ ramps) with 80 different frequencies and eight sound-pressure levels from 0 to $70 \mathrm{~dB}$ were delivered by an in-ear speaker. Following a craniotomy above the primary auditory cortex (AI) (Fig. 2a), the graphene array or penetrating electrodes were placed on the cortical surface or advanced to layer 4, respectively (Fig. 2b). We obtained local field potentials (LFPs) in response to different sounds and constructed caudal-rostral frequency maps (or tonotopic maps). The characteristic frequency (CF) was determined using the tip of a V-shaped tuning curve placed at each graphene/ penetrating-electrode site, which represents the LFP at the lowest sound intensity. The CF distribution over the AI showed the expected frequency organization.

The overall organization of the surface maps was similar to that of the maps assessed in layer 4 . However, differences in RF size, tuning-curve size, sound-evoked firing rate at the $\mathrm{CF}$, bandwidth (at $20 \mathrm{~dB}$ above the hearing threshold; BW20), and spike amplitude except corticalresponse threshold were observed between surface and penetrating recordings [Fig. 2c; all surface measures were normalized to layer 4 values. RF size: $387 \pm 47 \%$ ( $n=12$, $F=23.05, p<0.0001)$; tuning curve size: $139.9 \pm 3.9 \%(n$ $=68, F=48.43, p<0.0001)$; firing rate: $143.6 \pm 5.0 \%(n=$ $128, F=18.33, p<0.0001)$; spike amplitude: $78.7 \pm 1.9 \%$ 
a

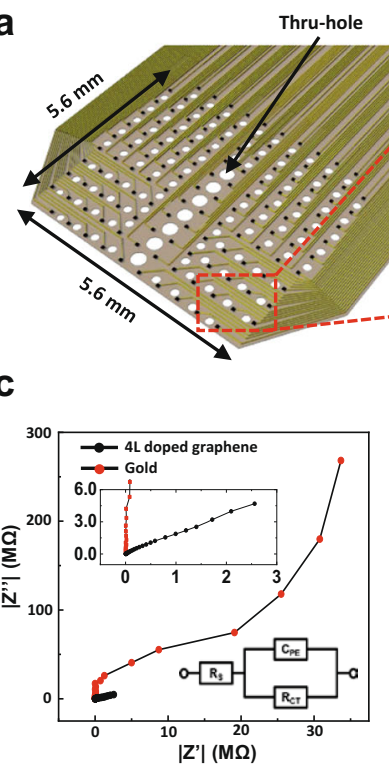

f

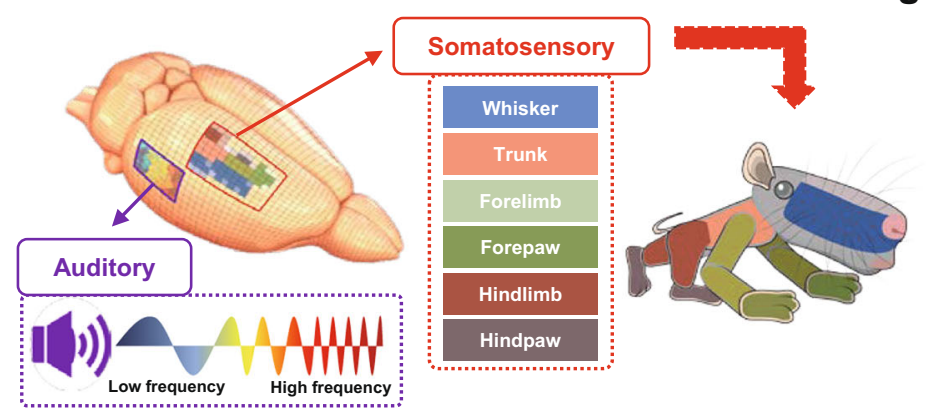

b

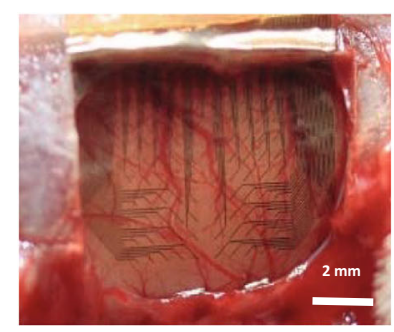

e

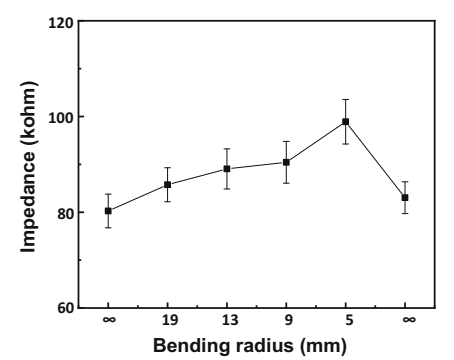

g

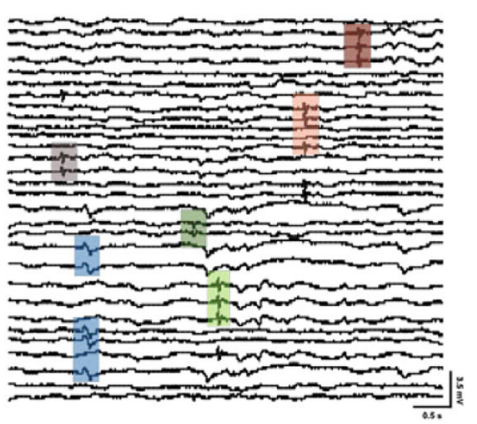

Fig. 1 Graphene-based microelectrode array for brain mapping on the cortical surface. a Schematic illustration of the graphene-based electrode array. $\mathbf{b}$ Optical image of the device on the cortical surface. c Nyquist plot of $4 \mathrm{~L}$-doped graphene and gold electrodes; measurements were conducted using the three-electrode method with a platinum counter electrode and $\mathrm{Ag} / \mathrm{AgCl}$ reference electrode in $0.01 \mathrm{M}$ phosphorus buffered saline. The surface area of the electrode is $60 \times 60 \mathrm{\mu m}^{2}$. A graph and an image of the inset show the magnified plot of $4 \mathrm{~L}$-doped graphene and gold electrodes and an equivalent circuit model of $4 \mathrm{~L}$-doped graphene and gold, respectively. The results of the equivalent circuit model are noted in Table S1. d Histogram showing the impedance of 30 channels comprising the electrode array. Impedance was derived via electrochemical impedance spectroscopy (EIS) at $1 \mathrm{kHz}$. e Impedance of $4 \mathrm{~L}$-doped graphene electrodes with respect to the bending radius. $\mathbf{f}$ Illustrations of rat auditory and somatosensory cortices. Two maps of auditory and somatosensory areas responding to auditory stimuli with various frequency tones and physical stimuli applied to each body part. $\mathbf{g}$ Thirty-channel (excluding the reference and ground electrodes) recording of the grapheneelectrode array on the cortical surface. The colors of boxes correlate with the geographical location of the rat's cortical surface.

$(n=511, F=120.0, p<0.0001) ; \mathrm{BW} 20: 132.8 \pm 3.3 \%(n=$ 68, $F=60.27, p<0.0001)$; and cortical threshold: $97.1 \pm$ $0.6 \%(n=68, F=3.17, p=0.077)]$. Moreover, no frequency dependence was observed for the altered responses across the tonotopic regions (Fig. S3).

The differences between surface and layer-4 recordings in the somatosensory cortex of the rats were similar to those in their primary AI. We also obtained recordings from superficial layer $1 / 2$ with penetrating electrodes in the somatosensory cortex. Somatosensory surface maps with graphene electrodes were compared with cortical layer $1 / 2$ and 4 maps assessed with penetrating electrodes.
LFPs for each sensory stimulus to the whisker, forepaw, forelimb, hind paw, hindlimb, trunk, etc., were located in the somatosensory cortex. Somatosensory maps were constructed using the LFP amplitude to produce a rodent homunculus (Fig. 3a).

We did not detect differences in the topographic organization of stimulus selectivity between the recording methodologies. However, the cortical-surface recordings showed weaker responses and a more expanded RF area than those obtained with penetrating recordings in the superficial layer $1 / 2$. These differences exceed the penetrating recordings in layer 4. [In Fig. 3b, c, all the measures 
a

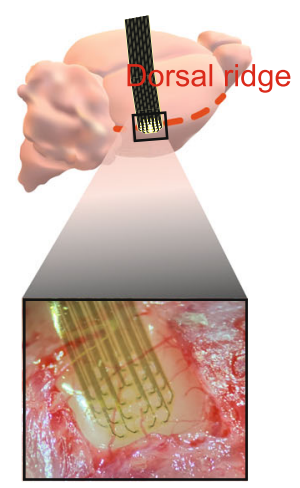

ci

$4.1 \mathrm{kHz}$

Surface
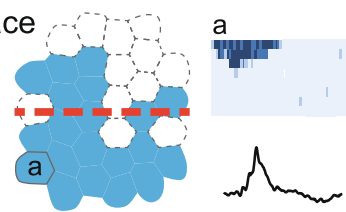

Layer 4

d
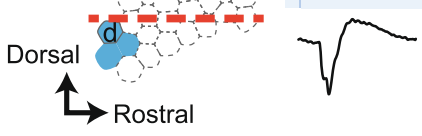

cii

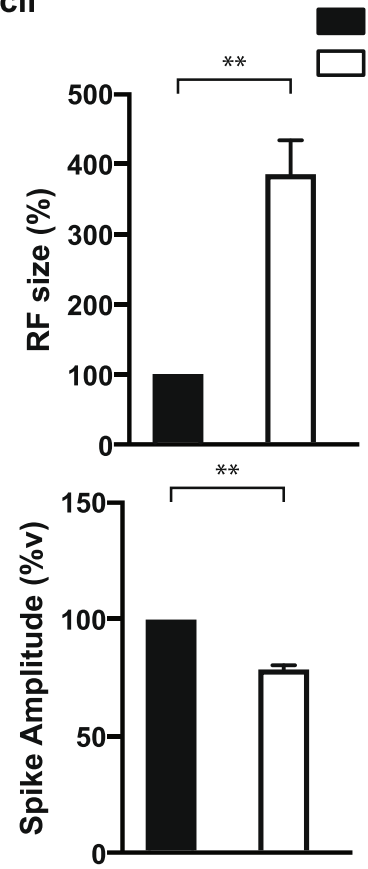

bi

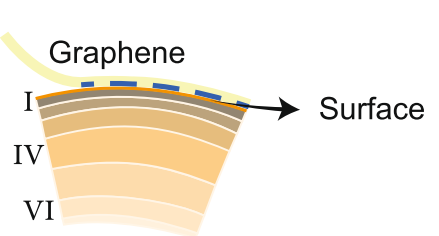

bii

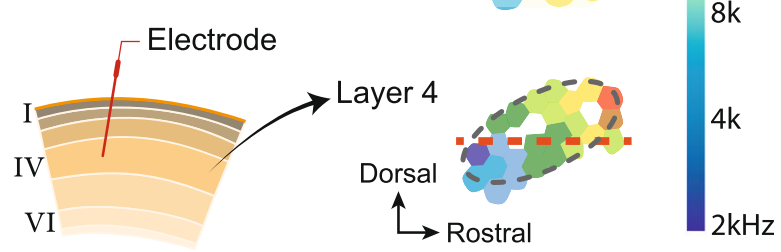

Tonotopic Map

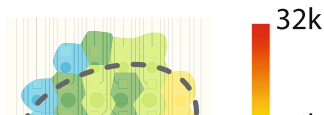

$16 \mathrm{k}$

$8 \mathrm{k}$

.

$2 \mathrm{kHz}$
$8.2 \mathrm{kHz}$

$16.4 \mathrm{kHz}$
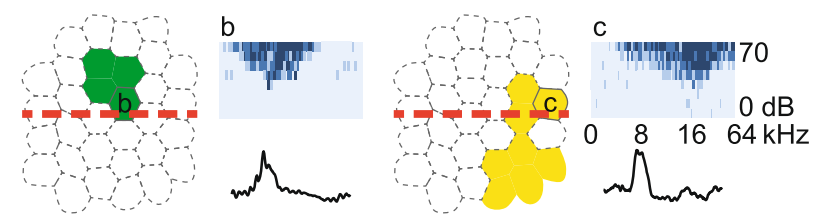

e
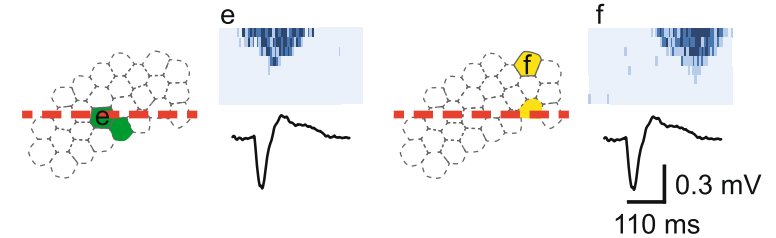

\section{Layer 4}

\section{Surface}
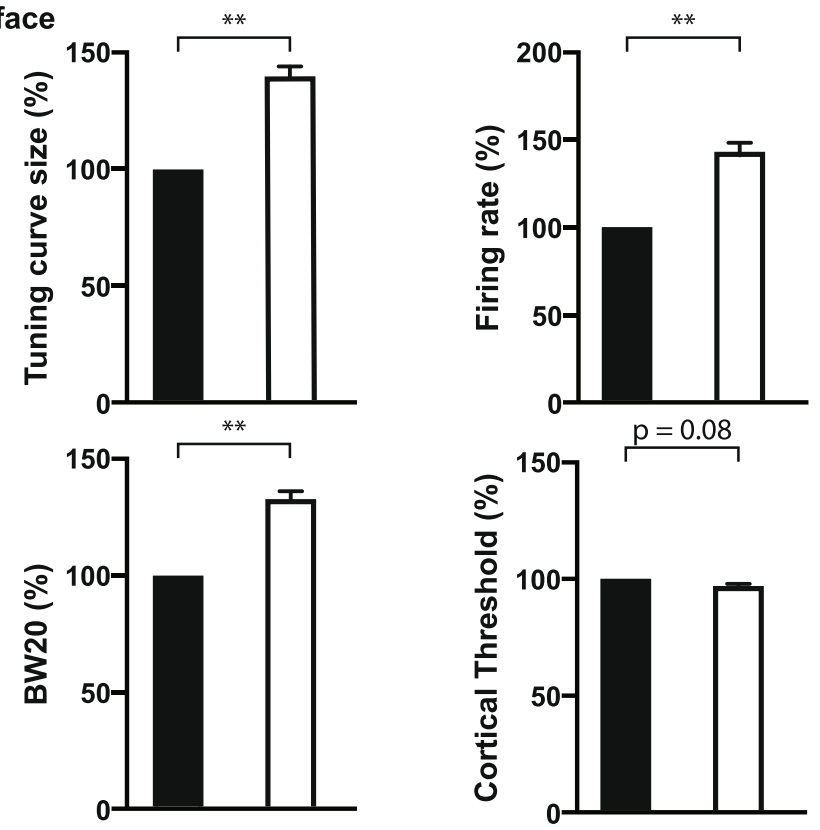

Fig. 2 Layer-specific auditory maps. a Graphene array applied to the cortical surface or layer 4 of the primary auditory cortex. $\mathbf{b}$ Tonotopic cortical maps generated in response to various frequencies. The dashed circle represents the putative primary auditory cortex (Al) in graphene-surface (bi) and penetrating layer-4 (bii) recordings. c Receptive fields (RFs), tuning curves, and LFPs are shown in response to 4.1, 8.2, and 16.4 kHz frequencies (ci). Cortical surface and layer-4 maps differ significantly in RF size, tuning-curve size, firing rate, spike amplitude, and BW20 but not in cortical threshold (cii). Data are presented as the mean \pm standard error (SEM). ${ }^{* *} p<0.01 ;{ }^{\text {n.s. }} p>0.05$. 

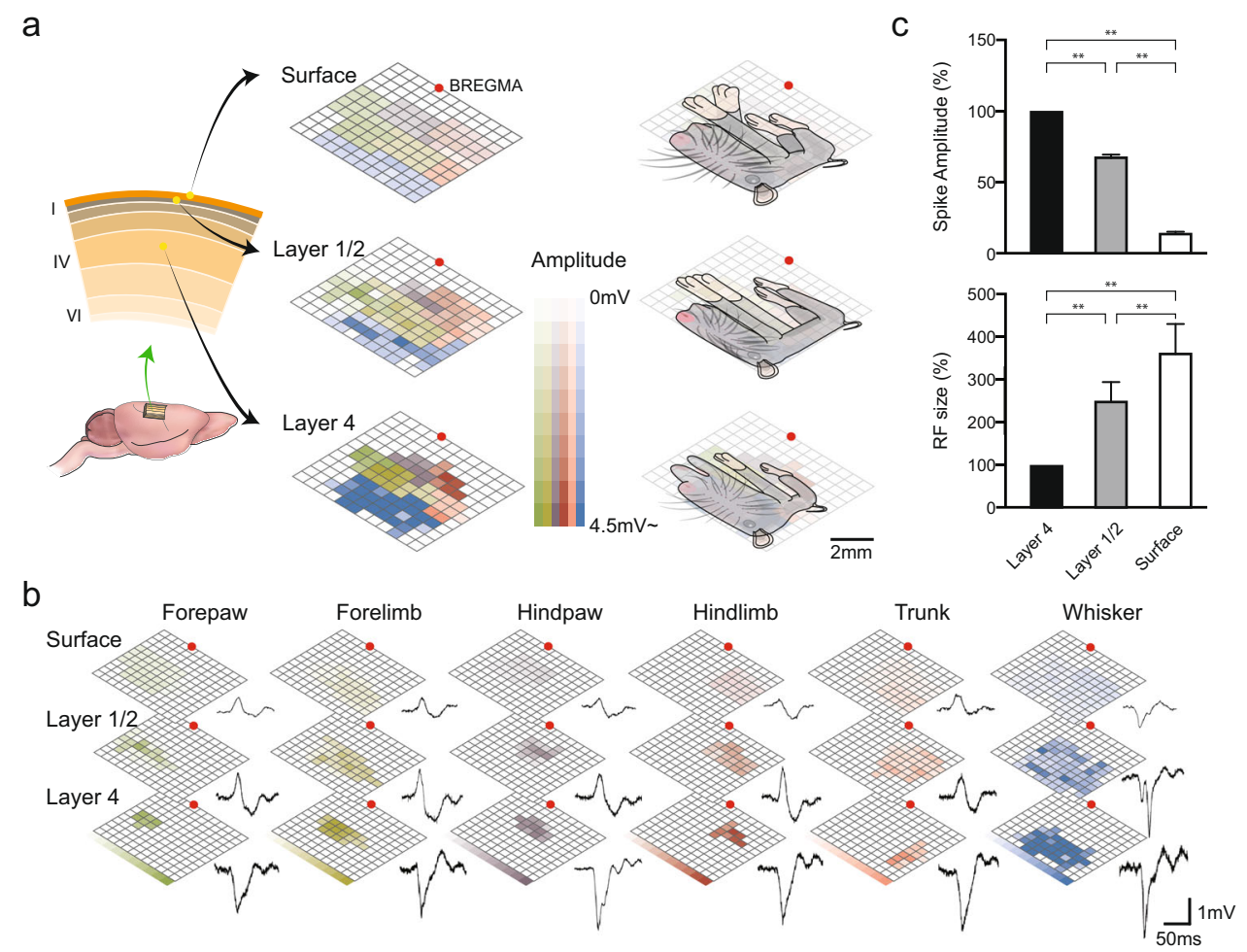

Fig. 3 Layer-specific somatosensory maps. a Location of neural recordings across cortical layers (left). Cortical surface maps determined using graphene electrodes were compared to cortical layer $1 / 2$, and four maps were produced using penetrating electrodes (middle). Color-coded somatosensory maps were constructed using the response amplitudes and overlapped with the homunculus (right). b Cortical responses and maps were recorded following the stimulation of each body part. Notably, the response amplitude and area stimulated by various somatosensory stimuli gradually decreased and expanded from layer 4 to layer $1 / 2$ and to the cortical surface, respectively. c Response amplitude and area across cortical layers were quantified by the size and location of LFPs. The area was normalized to the area recorded using graphene electrodes because of the different geographical locations of each somatosensory part. The response amplitude and area gradually decreased and expanded, respectively, as the electrodes were moved from cortical layer 4 to superficial layer $1 / 2$ and to the cortical surface. Cortical-surface recordings obtained using graphene arrays show weaker responses and less stimulus selectivity than those obtained with penetrating electrodes. Data are presented as the mean \pm standard error (SEM). ${ }^{* *} p<0.01 ;{ }^{\text {n.s. }} p>0.05$.

were normalized to layer- 4 values. Spike amplitude: layer $4(n=153)$ vs. layer $1 / 2(n=215), 68.0 \pm 0.97 \%$; layer $1 / 2$ vs. surface ( $n=1098), 14.4 \pm 0.17 \%$; layer 4 vs. surface; $F$ $=1265.5 ; p<0.0001$ for all comparisons. RF size: layer 4 vs. layer $1 / 2$, $250.7 \pm 44.0 \%$; layer $1 / 2$ vs. surface, $363.5 \pm$ $66.8 \%$; layer 4 vs. surface; $F=1265.5, n=6, p<0.0001$ for all comparisons.] The decreased selectivity from layer 4 to the superficial cortex was likely due to the distinct hierarchical structures of neural circuits from layer 4 to the surface, rather than the different recording materials, as responses in penetrating layer $1 / 2$ recordings became weaker and less selective when compared with those in penetrating layer 4 recordings.

Next, we examined whether graphene-based cortical surface stimulation could induce sensory map reorganization. Previous studies have shown that theta burst stimulation (TBS), a physiologically relevant high-frequency stimulation, is effective in inducing neuroplasticity and has therapeutic applications $^{24-26}$. The cortical-surface and layer 4 LFPs in response to sound stimuli were simultaneously monitored following the TBSs (Fig. 4a). Five successive TBSs spaced 10 min apart induced significant enhancements in RF size, tuning curve size, firing rate, spike amplitude, spike duration, and BW20 in both cortical-surface and layer-4 recordings without any observable frequency dependence of the altered responses (Fig. S4). [Fig. 4b; values were normalized to preTBS values. RF size: surface, $1558 \pm 263 \%(n=13, F=23.96$, $p<0.0001)$; tuning-curve size: layer $4,148.7 \pm 9.8 \%(n=44$, $F=13.37, p=0.0004)$ and surface, $204.3 \pm 5.3 \%(n=448$, $F=126.0, p<0.0001)$; firing rate: layer $4,204.2 \pm 13.8 \%$ $(n=44, F=15.72, p=0.0002)$ and surface, $198.5 \pm 7.9 \%$ ( $n=446, F=91.14, p<0.0001)$; spike amplitude: layer 4 , $134.3 \pm 4.9 \%(n=103, F=51.36, p<0.0001)$ and surface, $222.8 \pm 19.9 \%(n=61, F=47.56, p<0.0001)$; BW20: layer 4, $160.2 \pm 21.7 \% \quad(n=14, \quad F=3.59, \quad p=0.06)$ and surface, $154.1 \pm 5.7 \%(n=38, F=27.39, p<0.0001)]$. 
a

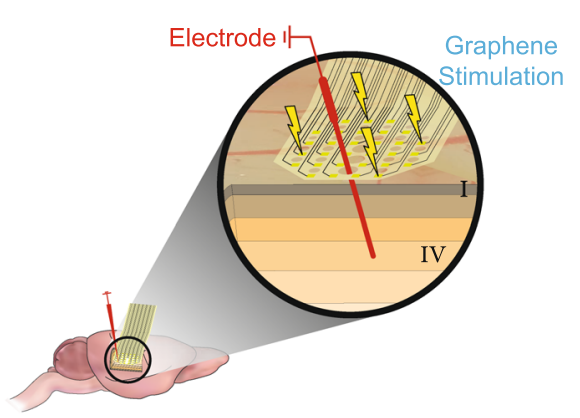

bii

Layer 4 Surface
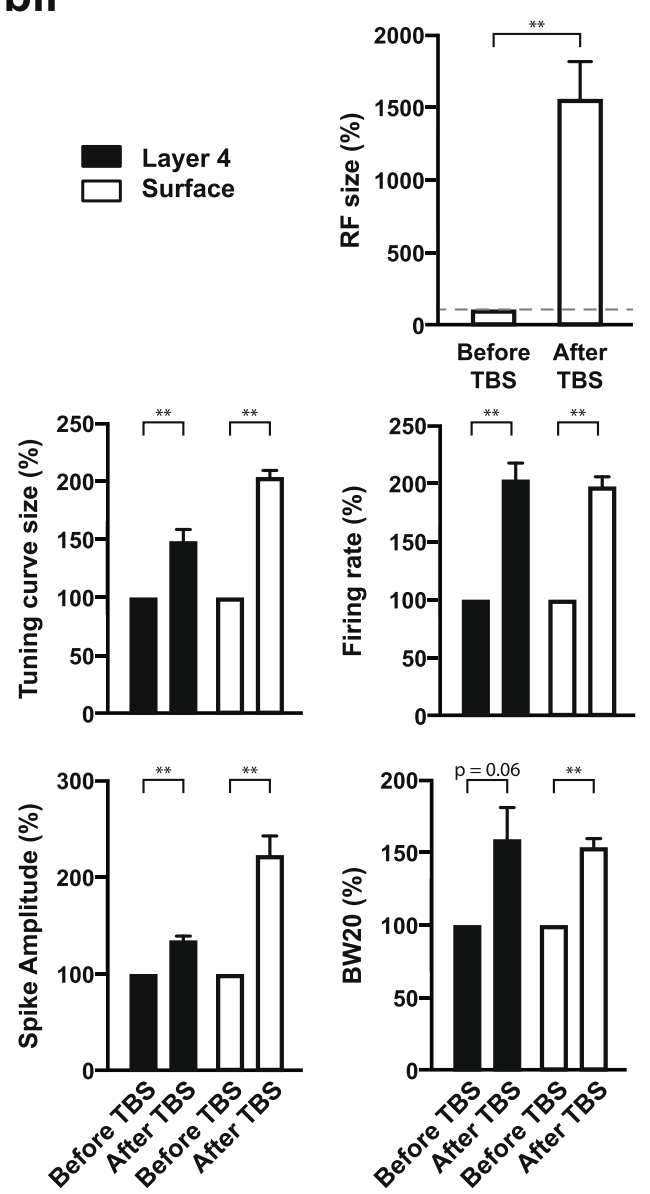

bi

\section{Surface recording}

Before TBS After TBS

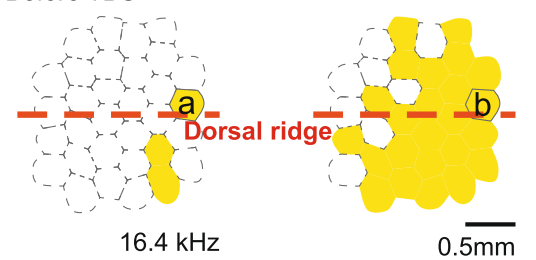

a

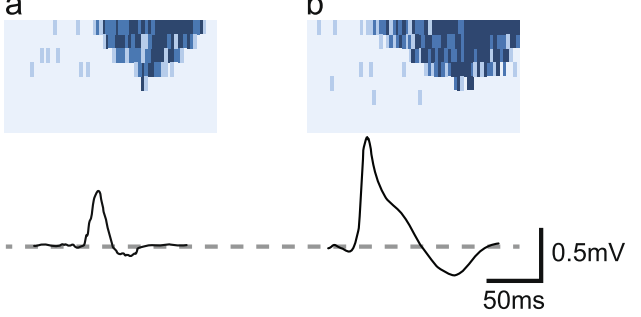

Layer 4 recording

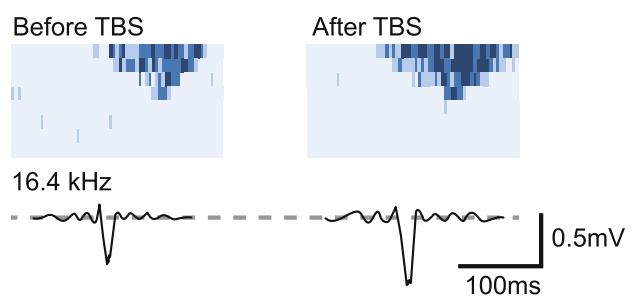

C

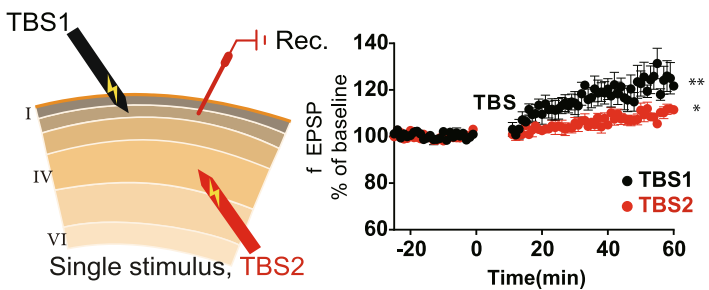

Time(min)

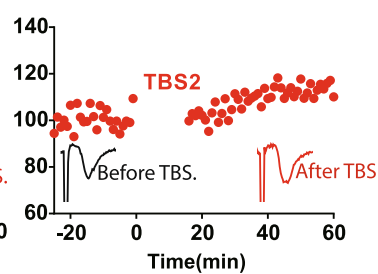

Fig. 4 Effects of graphene-based cortical stimulation on the map and synaptic plasticity. a Illustration of cortical stimulation at both the cortical surface and layer 4 using a graphene array. b Surface and layer-4 LFPs induced by auditory stimuli were simultaneously monitored before and after TBS at the cortical surface (bi). Cortical surface stimulation increased the RF size, tuning curve size, firing rate, spike amplitude, spike duration, and bandwidth (bii). c Using auditory brain slices, neural responses at the cortical surface were monitored using a stimulating electrode at cortical layer 4. TBSs were applied at two different locations: one stimulus (TBS1) at the cortical surface and the other (TBS2) at cortical layer 4. Although both TBS1 and TBS2 induced neural enhancement (bottom), the enhancement with TBS1 was larger than that with TBS2 (upper right). Data are presented as the mean \pm standard error (SEM). ${ }^{*} p<0.05 ;{ }^{* *} p<0.01$.

Furthermore, we examined whether Hebbian synaptic plasticity was involved in the observed map reorganization. In sectioned brains, neural responses were monitored at the cortical surface following electrical stimulation at cortical layer 4 . Once stable responses were detected, after $20 \mathrm{~min}$, four TBSs were applied at two different locations: one stimulus (TBS1) at the cortical surface and the other (TBS2) at cortical layer 4. These stimuli mimicked cortical-surface stimulation and sensory-driven input, respectively (Fig. 4c). Both TBS1 


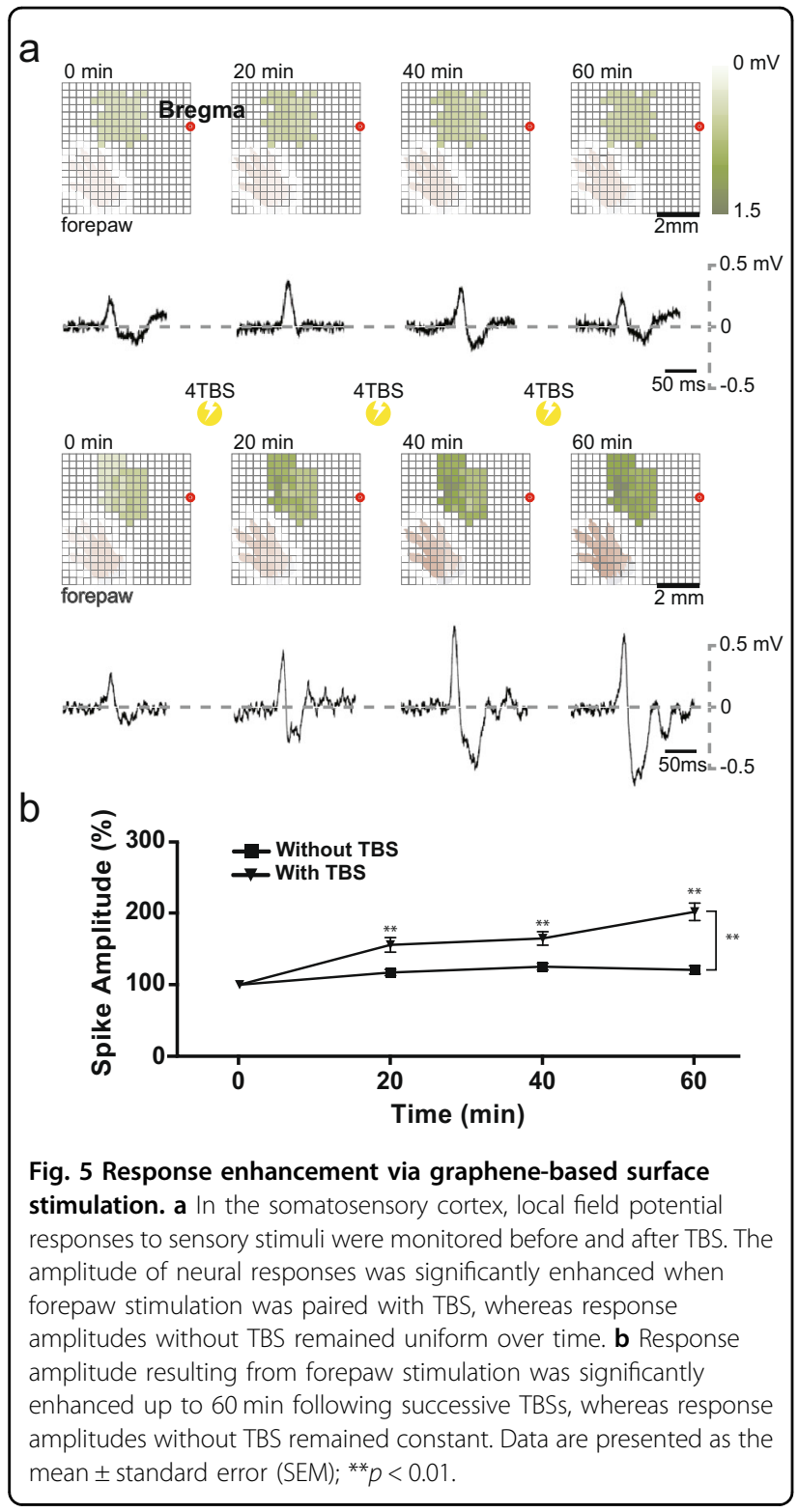

and TBS2 induced neural enhancement [TBS1, $121.7 \pm$ 6.7\% $(n=6, t=3.84, p=0.012)$; TBS2, $111.5 \pm 1.9 \%(n=$ $6, t=9.17, p=0.0003)]$. Interestingly, TBS1 produced a significantly larger enhancement $(F=5.14, p=0.046)$. This outcome indicated that, compared with deep stimulation, cortical-surface stimulation rendered cortical responses more susceptible to changes.

Cortical-surface stimulation-enhanced responses were similarly observed in the somatosensory cortex (Fig. 5). The LFP responses to somatosensory stimuli were monitored before and after the TBS. For example, forepaw stimulation-induced LFP deflections at several graphenerecording spots (Fig. 5a). The response amplitude resulting from forepaw stimulation was significantly enhanced up to $60 \mathrm{~min}$ following successive TBSs, while the response amplitude without the TBS remained constant. [Fig. 5b, before TBS vs. after TBS, $n=19,(20 \mathrm{~min})$ $155.8 \pm 17.1 \%$, (40 min) $164.8 \pm 15.1 \%$, (60 min) $202.1 \pm$ $20.5 \%$, paired $t$ test, $p<0.05$, and with TBS vs. without TBS, Fisher's PLSD, $F>23.27, p<0.0013$ in each comparison.] It is notable that the RF size was not studied because of the limited size of our graphene array. These results indicated that cortical surface stimulation induces remodeling of sensory maps along the columnar network.

\section{Discussion}

Existing tools for cortical mappings, such as tetrodes and glass pipettes, have limited clinical use because of their invasive nature, limiting repeated recordings and leading to high time consumption. Magnetic resonance imaging does not share these limitations but has poor temporal and spatial resolutions. Our recently developed graphene-based multichannel electrode array overcomes these limitations, provides technology that is less invasive, and offers high-throughput with high spatiotemporal resolution. This electrode array permits rapid assessment of cortical sensory maps and enables a simple workflow for assessing the macroscale plasticity of these maps.

Additionally, our findings can provide an important framework for understanding how neural signals are integrated into hierarchical networks from thalamorecipient layer 4 , a primary source of thalamic inputs, to the cortical surface, a center of heavy synaptic integration. Finally, the device has diagnostic and therapeutic applications for several brain map-related diseases.

In both auditory and somatosensory cortices, the overall organization in maps assessed at the surface with graphene electrodes was similar to those in maps assessed at layer 4 . However, surface maps exhibited more attenuated response amplitudes and more diffused stimulus selectivity than layer 4 maps. This outcome was likely due to variations in the columnar organization, as soma clusters are abundant in layer 4 whereas apical dendrites dominate the cortical surface. As signal decay is common along an apical dendrite from the soma where action potentials arise, on the apical tuft on the cortical surface, attenuated responses to sensory stimuli are expected in cortical-surface recordings.

The cortical surface receives crossmodal inputs and columnar inputs within one modality. In addition, the dendrites in an apical tuft have long horizontal projections and integrate heterogeneous inputs from many cortical areas. This dendritic organization permits some degree of overlap in multi-tuned inputs, thus enlarging the area of sensory representation ${ }^{27,28}$. Such network complexity seems to be required for multi-pitch perception, perceptual learning, information shaping, working memory, feature binding, and multisensory integration ${ }^{29-34}$. As sensory inputs recorded at the cortical surface are not homogeneous or 
concentrated, cortical-surface responses can have broader RFs than other responses while retaining significant information regarding sensory integration.

Our data verify that plasticity at the synapse and sensory map levels can be induced via graphene stimulation of the cortical surface. The types of neuromodulators that are involved in this plasticity remain unclear. Neuromodulation evokes progressive changes in the amplitude and selectivity of cortical responses, thereby shaping cortical sensory representations. For example, activating the nucleus basalis (known as a major source of cholinergic intervention) alters the firing rates and patterns of cells ${ }^{35-39}$. Neuromodulatory innervation can induce Hebbian and non-Hebbian synaptic plasticity even after the critical period ${ }^{11,36,40-42}$. Synchronized stimulation of dopaminergic synapses to specific tones increases cortical responses to the stimulated frequencies and increases the area of the tonotopic map that is dedicated to that frequency ${ }^{41,43}$.

Neuromodulatory afferents are located primarily in dendrites that project to the cortical surface, which is near our electrical stimulation; our studies also revealed the central role of neuromodulation in cortical plasticity. In two-monthold rats experiencing closure of the critical period, graphenebased cortical-surface stimulation could promote both synaptic and map plasticity in centimeter-scale areas. Our results clearly verified that graphene stimulation at the cortical surface lowered the threshold for sensory responses, suggesting perceptual enhancement, which leads to functional recovery from sensory deprivation.

\section{Experimental methods}

\section{Graphene-electrode fabrication}

Monolayer graphene was grown via the typical CVD method on a $\mathrm{Cu}$ foil and was multistacked in four layers (4 L) using conventional stacking before chemical doping. Polyimide (PI) with a thickness of $1.2 \mu \mathrm{m}$ was spin-coated onto a $\mathrm{Cu}$ carrier substrate. $\mathrm{Cr} / \mathrm{Au}(3 \mathrm{~nm} / 40 \mathrm{~nm})$ interconnects were formed on the PI film via thermal evaporation and photolithography. Four-layer graphene was transferred onto the PI substrate in contact with the metal Au-tracking material and patterned using photolithography and oxygen plasma etching. The graphene area for each contact electrode $\left(60 \times 60 \mu \mathrm{m}^{2}\right)$ was isolated via photolithography and oxygen-plasma etching and interconnected with gold metal lines. Then, the entire area, except for the graphene-contact electrode, was covered with SU-8 photoresist for insulation.

\section{Animal preparation}

C57BL/6J mice (for auditory maps) and Sprague-Dawley (SD) rats (for somatosensory maps), aged 8-12 weeks, were housed under a 12-h light/dark cycle and provided with food and water ad libitum. Ketamine and xylazine $(90 \mathrm{mg} / \mathrm{kg}$ and $10 \mathrm{mg} / \mathrm{kg}$, intraperitoneal) were used to anesthetize 8-12- week-old mice with their heads fixed in a stereotaxic apparatus. The body temperature $\left(\sim 36.5^{\circ} \mathrm{C}\right)$ and physiological state (respiratory rate, heart rate, corneal reflex, and hind paw reflex) were continuously monitored while the mice were under anesthesia. Cerebral spinal fluid (CSF) was drained to reduce the likelihood of cerebral edema.

The muscles were blunt-dissected over the occipital skull to expose the cisterna magna above the axis vertebra at the top of the spinal cord. Craniotomy was performed over the parietal cortex to expose the somatosensory cortex and over the temporal cortex to expose the AI. The dura was subsequently slit with a sharp knife, and a cotton wick was used to drain the CSF. Then, a graphene array and/or penetrating tungsten tetrode were/was applied to the cortical surface and/or layer 4 .

All animal-handling procedures were approved by the Institutional Animal Care and Use Committee of the City University of Hong Kong (A-0117) and Incheon National University (INU-ANIM-2017-08). This research complies with all requirements, according to the guidelines of our institution's animal welfare committee and according to the journal's ethics policies.

\section{Somatosensory mapping}

Neuronal activity was recorded using both a penetrating electrode and a graphene-based electrode array. For the reconstruction of somatosensory maps, neural signals were detected following the stimulation of various body parts, including the whisker, forepaw, forelimb, hind paw, hindlimb, and trunk. To evoke sensory responses in the somatosensory cortex, two types of cutaneous stimuli were used, depending on the region being stimulated. Body parts were stimulated by a punctate touch with a wooden stick, whereas the whiskers were stimulated by bending stress that was applied with a fine stick.

A graphene-based electrode array and a sharp electrode were implanted at the cortical surface and into the deep layers of the cortex, respectively, to record the responses evoked by mechanical stimulation. The grapheneelectrode recordings were amplified with a multichannel system using an RHD2000-series amplifier (RHD2000series amplifier evaluation system, Intan Technologies). The electrode array was connected to a flexible printed circuit board (FPCB) by bonding to an anisotropic conductive film (ACF). Next, the FPCB was joined to the amplifier board via a zero-insertion-force connector, and the amplifier board was connected to an RHD2000 USB interface board. Finally, the RHD2000 board was used to measure the brain responses recorded by the multichannel electrode array using proprietary software.

\section{Auditory mapping}

The procedure for auditory mapping has been detailed previously ${ }^{5,44,45}$. Neuronal activity, in response to various 
sound stimuli, was recorded using a penetrating electrode or graphene-based electrode array. To reconstruct the frequency-intensity RFs, we presented 50-ms tone pips (5$\mathrm{ms}$ cosine squared ramp) at 80 different frequencies $(0.4-63.5 \mathrm{kHz}, 0.1$-octave spacing) and eight soundpressure levels $(0-70 \mathrm{~dB}$ SPL in steps of $10 \mathrm{~dB})$. The CF was determined to be the frequency that induced a response at the lowest sound intensity, typically corresponding to the tip of a V-shaped RF.

The RF size was calculated based on the number of recording sites that responded to each frequency tone. The tuning curve size was calculated using the response size of a recording spot in response to various frequency sounds. The firing rate was calculated using the number of responses to the CF tones. BW20 was defined as the bandwidth $20 \mathrm{~dB}$ above the threshold of the tuning curve. The cortical threshold was measured at the lowest sound intensity for which cortical spikes were induced. Cortical tonotopic maps were reconstructed using Voronoi tessellation (Python). To induce map reorganization, TBS (10 sets of four burst pulses at $100 \mathrm{~Hz}$ with a $200-\mathrm{ms}$ interburst interval) was applied to the cortical surface five times, with $10 \mathrm{~min}$ between all rounds of the TBS.

\section{Cortical slice preparation and long-term potentiation (LTP) induction}

The procedures for brain preparation and electrophysiology have been detailed previously ${ }^{5}$. To acquire brain slices, rats (aged 4-6 weeks) were deeply anesthetized with $2 \%$ isoflurane. The brain was quickly removed and placed into a chilled $\left(4{ }^{\circ} \mathrm{C}\right)$, oxygenated $\left(5 \% \mathrm{CO}_{2}\right.$ and $95 \% \mathrm{O}_{2}$ ) slicing medium containing $212 \mathrm{mM}$ sucrose, $5 \mathrm{mM} \mathrm{KCl}, 1.23 \mathrm{mM} \mathrm{NaH}_{2} \mathrm{PO}_{4}, 26 \mathrm{mM} \mathrm{NaHCO} 3,11 \mathrm{mM}$ glucose, $1.5 \mathrm{mM} \mathrm{MgCl}_{2}$, and $2.5 \mathrm{mM} \mathrm{CaCl}$. Auditory brain slices $(400 \mu \mathrm{m})$ were cut and then transferred to a holding chamber containing oxygenated physiological saline $\left(124 \mathrm{mM} \mathrm{NaCl}, 4 \mathrm{mM} \mathrm{KCl}, 1.23 \mathrm{mM} \mathrm{NaH} \mathrm{PO}_{4}\right.$, $26 \mathrm{mM} \mathrm{NaHCO}, 10 \mathrm{mM}$ glucose, $1.5 \mathrm{mM} \mathrm{MgCl}_{2}$, and $2 \mathrm{mM} \mathrm{CaCl} 2$ ).

After $\sim 1 \mathrm{~h}$ of recovery, individual slices were transferred to a recording chamber and continuously superfused with oxygenated physiological saline $(1.5 \mathrm{ml} / \mathrm{min})$. LTP was induced using a response amplitude that elicited $75 \%$ of the maximal local field response observed during TBS. The slopes of the evoked field excitatory postsynaptic potentials were measured and expressed relative to the normalized preconditioned baseline. The response change was measured as a percentage of the baseline slope.

\section{Statistics}

Statistical differences were evaluated using one-way analysis of variance and post hoc Fisher's test was used to predict the least-significant differences between groups. Paired $t$ tests was used to predict the least-significant differences within groups (with StatView or SAS statistical analysis software); the significance level was set as * $p<$ $0.05,{ }^{* * *} p<0.01,{ }^{\text {n.s. }} p>0.05$. Data are presented as the mean \pm standard error and normalized for comparisons.

\section{Acknowledgements}

This work was supported by the Technology Innovation Program (20012355, fully implantable closed-loop Brain to $X$ for voice communication) funded by MOTIE, Korea and High Risk, High Return Research Program (2020) in the Incheon National University for Sunggu Yang and the National Research Foundation of Korea (NRF-2015R1A3A2066337) for J.-H Ahn. Sungchil Yang acknowledged grants from the Research Grants Council of Hong Kong (11102120 and 11102618).

\section{Author details \\ 'Department of Neuroscience, City University of Hong Kong, Tat Chee Avenue, Kowloon, Hong Kong. ${ }^{2}$ Department of Nanobioengineering, Incheon National University, Incheon 22012, Republic of Korea. ${ }^{3}$ gBrain Inc., Incheon 21984 Republic of Korea. ${ }^{4}$ School of Electrical \& Electronic Engineering, Yonsei University, Seoul 03722, Republic of Korea. ${ }^{5}$ Neuroscience Graduate Program, University of Arizona, Tucson, AZ 85721, USA. ${ }^{6}$ Department of Physiology, University of Arizona, Tucson, AZ 85724, USA}

\section{Author contributions}

M.L., S.L., and J.K. contributed equally to the work. J.K. and J.S.L. designed and tested the devices. M.L., S.L., J.H.L., and S.M. performed brain mapping. M.L., S.L., J.K., and J.H.L. analyzed the data. J.H.A., S.G.Y., and S.C.Y. designed the experiments and wrote the paper. S.M., S.B., J.H.A., S.G.Y., and S.C.Y. edited and revised the paper.

Conflict of interest

The authors declare no competing interests.

\section{Publisher's note}

Springer Nature remains neutral with regard to jurisdictional claims in published maps and institutional affiliations.

Supplementary information The online version contains supplementary material available at https://doi.org/10.1038/s41427-021-00334-8.

Received: 15 July 2021 Revised: 12 August 2021 Accepted: 23 August 2021. Published online: 1 October 2021

\section{References}

1. Feldman, D. E. Synaptic mechanisms for plasticity in neocortex. Annu. Rev. Neurosci. 32, 33-55 (2009).

2. Tao, H. W., Li, Y. T. \& Zhang, L. I. Formation of excitation-inhibition balance: inhibition listens and changes its tune. Trends Neurosci. 37, 528-530 (2014)

3. Williamson, R. S., Hancock, K. E., Shinn-Cunningham, B. G. \& Polley, D. B. Locomotion and Task Demands Differentially Modulate Thalamic Audiovisual Processing during Active Search. Curr. Biol. 25, 1885-1891 (2015).

4. Hensch, T. K. Critical period plasticity in local cortical circuits. Nat. Rev. Neurosci. 6, 877-888 (2005)

5. Yang, S., Weiner, B. D., Zhang, L. S., Cho, S. J. \& Bao, S. Homeostatic plasticity drives tinnitus perception in an animal model. Proc. Natl Acad. Sci. USA. 108, 14974-14979 (2011).

6. Espinosa, J. S. \& Stryker, M. P. Development and plasticity of the primary visual cortex. Neuron 75, 230-249 (2012)

7. Han, Y. K., Kover, H., Insanally, M. N., Semerdjian, J. H. \& Bao, S. Early experience impairs perceptual discrimination. Nat. Neurosci. 10,1191-1197 (2007).

8. Yang, S., Zhang, L. S., Gibboni, R., Weiner, B. \& Bao, S. Impaired development and competitive refinement of the cortical frequency map in tumor necrosis factor-a-deficient mice. Cereb. Cortex 24, 1956-1965 (2014).

9. Masri, S. et al. Blast Exposure Disrupts the Tonotopic Frequency Map in the Primary Auditory Cortex. Neuroscience 379, 428-434 (2018). 
10. Barkat, T. R., Polley, D. B. \& Hensch, T. K. A critical period for auditory thalamocortical connectivity. Nat. Neurosci. 14, 1189-1194 (2011).

11. Buonomano, D. V. \& Merzenich, M. M. Cortical plasticity: from synapses to maps. Annu. Rev. Neurosci. 21, 149-186 (1998).

12. Yang, S., Zhang, L. S., Gibboni, R., Weiner, B. \& Bao, S. Impaired development and competitive refinement of the cortical frequency map in tumor necrosis factor-alpha-deficient mice. Cereb. Cortex 24, 1956-1965 (2014).

13. Stensaas, S. S. \& Stensaas, L. J. Histopathological evaluation of materials implanted in the cerebral cortex. Acta Neuropathol. 41, 145-155 (1978).

14. Hofmeijer, J. C. et al. Detecting Cortical Spreading Depolarization with Full Band Scalp Electroencephalography: An Illusion? Front. Neurol. 9, 17 (2018).

15. Dreier, J. P. et al. Recording, analysis, and interpretation of spreading depolarizations in neurointensive care: Review and recommendations of the COSBID research group. J. Cereb. Blood Flow. Metab. 37, 1595-1625 (2017).

16. Rickard, T. C. et al. The calculating brain: an fMRI study. Neuropsychologia 38, 325-335 (2000).

17. Deneux, T. et al. Accurate spike estimation from noisy calcium signals for ultrafast three-dimensional imaging of large neuronal populations in vivo. Nat. Commun. 7, 12190 (2016).

18. Lee, J. H. et al. Global and local fMRI signals driven by neurons defined optogenetically by type and wiring. Nature 465, 788-792 (2010).

19. Park, D. W. et al. Graphene-based carbon-layered electrode array technology for neural imaging and optogenetic applications. Nat. Commun. 5, 5258 (2014).

20. Kuzum, D. et al. Transparent and flexible low noise graphene electrodes for simultaneous electrophysiology and neuroimaging. Nat. Commun. 5, 5259 (2014).

21. Viventi, J. et al. Flexible, foldable, actively multiplexed, high-density electrode array for mapping brain activity in vivo. Nat. Neurosci. 14, 1599-1605 (2011).

22. Masvidal-Codina, E. et al. High-resolution mapping of infraslow cortical brain activity enabled by graphene microtransistors. Nat. Mater. 18, 280-288 (2019).

23. Blaschke, B. M. et al. Mapping brain activity with flexible graphene microtransistors. 2d Mater. 4, 025040 (2017).

24. Park, S.-W. et al. Epidural Electrotherapy for Epilepsy. Small 14, e1801732 (2018).

25. Lian, J., Bikson, M., Sciortino, C., Stacey, W. C. \& Durand, D. M. Local suppression of epileptiform activity by electrical stimulation in rat hippocampus in vitro. J. Physiol. 547, 427-434 (2013).

26. Jensen, A. L. \& Durand, D. M. Suppression of axonal conduction by sinusoidal stimulation in rat hippocampus in vitro. J. Neural Eng. 4, 1-16 (2007).

27. Brown, S. P. \& Hestrin, S. Intracortical circuits of pyramidal neurons reflect their long-range axonal targets. Nature 457, 1133-1136 (2009).

28. Spruston, N. Pyramidal neurons: dendritic structure and synaptic integration. Nat. Rev. Neurosci. 9, 206-221 (2008).
29. Petrus, E. et al. Crossmodal induction of thalamocortical potentiation leads to enhanced information processing in the auditory cortex. Neuron 81, 664-673 (2014).

30. Oswald, A. M. \& Reyes, A. D. Maturation of intrinsic and synaptic properties of layer 2/3 pyramidal neurons in mouse auditory cortex. J. Neurophysiol. 99, 2998-3008 (2008).

31. Chen, $X$. et al. Encoding and retrieval of artificial visuoauditory memory traces in the auditory cortex requires the entorhinal cortex. J. Neurosci. 33, 9963-9974 (2013).

32. Beierholm, U. R. et al. Bayesian priors are encoded independently from likelihoods in human multisensory perception. J. Vis. 9, 21-29 (2009).

33. Kampa, B. M., Letzkus, J. J. \& Stuart, G. J. Cortical feed-forward networks for binding different streams of sensory information. Nat. Neurosci. 9, 1472-1473 (2006).

34. Kozloski, J., Hamzei-Sichani, F. \& Yuste, R. Stereotyped position of local synaptic targets in neocortex. Science 293, 868-872 (2001).

35. Kilgard, M. P. \& Merzenich, M. M. Cortical map reorganization enabled by nucleus basalis activity. Science 279, 1714-1718 (1998).

36. Weinberger, N. M. Specific long-term memory traces in primary auditory cortex. Nat. Rev. Neurosci. 5, 279-290 (2004)

37. Yu, A. J. \& Dayan, P. Uncertainty, neuromodulation, and attention. Neuron 46 681-692 (2005)

38. Froemke, R. C., Merzenich, M. M. \& Schreiner, C. E. A synaptic memory trace for cortical receptive field plasticity. Nature 450, 425-429 (2007).

39. Sarter, M., Hasselmo, M. E., Bruno, J. P. \& Givens, B. Unraveling the attentional functions of cortical cholinergic inputs: interactions between signal-driven and cognitive modulation of signal detection. Brain Res. Brain Res. Rev. 48, 98-111 (2005).

40. Malenka, R. C. \& Bear, M. F. LTP and LTD: an embarrassment of riches. Neuron 44, 5-21 (2004)

41. Froemke, R. C. Plasticity of cortical excitatory-inhibitory balance. Annu. Rev. Neurosci. 38, 195-219 (2015).

42. Blundon, J. A. \& Zakharenko, S. S. Presynaptic gating of postsynaptic synaptic plasticity: a plasticity filter in the adult auditory cortex. Neuroscientist 19, 465-478 (2013).

43. Bao, S., Chan, V. T. \& Merzenich, M. M. Cortical remodelling induced by activity of ventral tegmental dopamine neurons. Nature 412, 79-83 (2001).

44. Miyakawa, A. et al. Tinnitus Correlates with Downregulation of Cortical Glutamate Decarboxylase 65 Expression But Not Auditory Cortical Map Reorganization. J. Neurosci. 39, 9989-10001 (2019).

45. Yang, S., Su, W. \& Bao, S. Long-term, but not transient, threshold shifts alter the morphology and increase the excitability of cortical pyramidal neurons. J. Neurophysiol. 108, 1567-1574 (2012). 